\title{
Mesoporous calcium-silicon xerogels with mesopore size and pore volume influence hMSC behaviors by load and sustained release of rhBMP-2
}

\author{
This article was published in the following Dove Press journal: \\ International Journal of Nanomedicine \\ 4 March 2015 \\ Number of times this article has been viewed
}

\author{
Wenhua Songl,* \\ Xiangde $\mathrm{Li}^{1}$,* \\ Jun Qian' \\ Guoyu Lv ${ }^{2}$ \\ Yonggang $\mathrm{Yan}^{2}$ \\ Jiacan $\mathrm{Su}^{3}$ \\ Jie Wei'
}

'Key Laboratory for Ultrafine Materials of Ministry of Education, East China University of Science and Technology, Shanghai, People's Republic of China; ${ }^{2}$ College of Physical Science and Technology, Sichuan University, Chengdu, People's Republic of China; ${ }^{3}$ Changhai Hospital, Second Military Medical University, Shanghai, People's Republic of China

*These authors contributed equally to this paper
Correspondence: Guoyu Lv; Jie Wei Sichuan University, No 24 South Section I, Yihuan Road, Chengdu 610065, People's Republic of China; East China University of Science and Technology, I 30 Meilong Road, Shanghai 200237,

People's Republic of China

Tel +862164251308

Fax +862164251358

Emailnic7505@263.net; jiewei7860@sina.com

\begin{abstract}
Mesoporous calcium-silicon xerogels with a pore size of $15 \mathrm{~nm}$ (MCS-15) and pore volume of $1.43 \mathrm{~cm}^{3} / \mathrm{g}$ were synthesized by using 1,3,5-mesitylene (TMB) as the poreexpanding agent. The MCS-15 exhibited good degradability with the weight loss of $50 \mathrm{wt} \%$ after soaking in Tris- $\mathrm{HCl}$ solution for 56 days, which was higher than the $30 \mathrm{wt} \%$ loss shown by mesoporous calcium-silicon xerogels with a pore size of $4 \mathrm{~nm}$ (MCS-4). The pore size and pore volume of MCS-15 had significant influences on load and release of recombinant human bone morphogenetic protein-2 (rhBMP-2). The MCS-15 had a higher capacity to encapsulate a large amount of rhBMP-2; it could adsorb $45 \mathrm{mg} / \mathrm{g}$ of rhBMP-2 in phosphate-buffered saline after 24 hours, which was more than twice that with MCS-4 $(20 \mathrm{mg} / \mathrm{g})$. Moreover, the MCS-15 system exhibited sustained release of rhBMP-2 as compared with MCS-4 system (showing a burst release). The MCS-15/rhBMP-2 system could promote the proliferation and differentiation of human mesenchymal stem cells, showing good cytocompatibility and bioactivity. The results indicated that MCS-15, with larger mesopore size and higher pore volume, might be a promising carrier for loading and sustained release of rhBMP-2, which could be used as bone repair material with built-in osteoinduction function in bone reconstruction.
\end{abstract}

Keywords: mesoporous calcium-silicon xerogels, pore size, pore volume, load-release, rhBMP-2

\section{Introduction}

Mesoporous materials with pores ranging in size from 2 to $50 \mathrm{~nm}$ have received enormous attention owing to their potential applications in catalysis, separation, sensing, drug delivery, enzyme immobilization, and so on. ${ }^{1-2}$ So far, some studies have reported the design of drug delivery systems based on the mesoporous materials for therapeutic molecules, including chemical drugs, peptides and proteins, and so on. ${ }^{3-5}$ As an effective molecule-delivering vector, the mesoporous materials with the pore size of a few nanometers have a high capacity to encapsulate large amounts of molecules. ${ }^{4,6}$ Moreover, the pore size, pore volume, and microstructure of the mesoporous materials can be adjusted according to the size and performances of delivered molecules. ${ }^{6,7}$ Recently, the application of mesoporous materials for bone regeneration has been proposed, and the tunable pore size and high pore volume of the mesoporous materials might allow them to load osteogenic agents and promote new bone tissue regeneration. ${ }^{6,8}$

The biomacromolecule bone morphogenetic protein (BMP) with strong boneinducing activity has been considered to play important roles in bone regeneration. ${ }^{9-11}$ Furthermore, recombinant human bone morphogenetic protein-2 (rhBMP-2) has 
received US Food and Drug Administration approval for application in the enhancement of lumbar spine fusion and treatment of acute tibial fractures. ${ }^{12}$ However, the bioactivity of BMP is deteriorated by its short in vivo half-life (BMP can be quickly diluted and metabolized). Therefore, a suitable carrier is required for preventing BMP from degradation and preserving its bioactivity in bone defects. A product which can deliver rhBMP-2 in a collagen matrix for release and improve local retention has been successfully applied in some clinical bone repair cases. However, the osteogenic outcome was limited by the rhBMP-2 release properties in the early period, which might not be sufficient to form the amount of bone needed to meet clinical demands. ${ }^{13,14}$ Therefore, a more effective rhBMP-2 delivery system should be developed.

Depending on the size of the delivering molecule, the pore size of the delivering vector has to be tuned to capture the molecule. Generally, small-molecule drugs are possibly loaded into the mesopore materials within the mesopores (around $2 \mathrm{~nm}$ ), while enlargement of mesopore size is often needed to deliver biomacromolecules such as proteins. Taking these facts into consideration, we designed a mesoporous biomaterial with larger mesopore size and higher pore volume for the delivery of the biomacromolecule rhBMP-2, which might offer the possibility of adsorbing or entrapping rhBMP-2 within the mesopores. Such mesoporous materials with improved diffusion of the guest macromolecules through mesoporous channels allow better macromolecule accessibility. Therefore, in this study, mesoporous calcium-silicon xerogels with a larger mesopore size of $15 \mathrm{~nm}$ (MCS-15) and pore volume of $1.43 \mathrm{~cm}^{3} / \mathrm{g}$ were prepared, and the influences of the mesopore size and pores volume of MCS-15 on rhBMP-2 loading and release were investigated. Moreover, the effects of MCS-15 loading rhBMP-2 on proliferation and differentiation of human mesenchymal stem cells (hMSCs) were also evaluated in vitro.

\section{Materials and methods Preparation of MCS-I5}

The MCS-15 was synthesized using the template induced method. The nonionic block copolymer EO20PO70EO20 (Pluronic P123, $M_{\mathrm{w}}=5,800$, Sigma-Aldrich Co., St Louis, MO, USA) and 3,5-mesitylene (TMB; Sinopharm Chemical Reagent Co., Ltd., Shanghai, People's Republic of China) were employed as template agents. In brief, $4.8 \mathrm{~g}$ of P123 was dissolved in $480 \mathrm{~mL}$ of $0.1 \mathrm{M} \mathrm{HCl}$ aqueous solution, and $6 \mathrm{~g}$ of TMB was slowly added into the solution at $45^{\circ} \mathrm{C}$ to obtain a white emulsion. Tetraethyl orthosilicon (Ling Feng Chemical Reagent Co., Ltd., Shanghai, People's Republic of China) and $\mathrm{Ca}\left(\mathrm{NO}_{3}\right)_{2} \cdot 4 \mathrm{H}_{2} \mathrm{O}(3.75 \mathrm{~g})$ were then added successively into the above suspension and stirred vigorously for 24 hours. After that, the reaction system was adjusted to $\mathrm{pH} 10$ using ammonia and placed into an oven at $100^{\circ} \mathrm{C}$ for 48 hours. The white precipitate was collected by filtration and then washed with ethanol and deionized water, which was then dried in an oven at $80^{\circ} \mathrm{C}$ for 12 hours. The final product was obtained after calcination in air at $550^{\circ} \mathrm{C}$ for 6 hours at a ramping rate of $5^{\circ} \mathrm{C} \mathrm{min}^{-1}$. The schematic diagram of the preparation process is shown in Figure 1A. MCS-4 was prepared as a control using the same procedure without TMB.
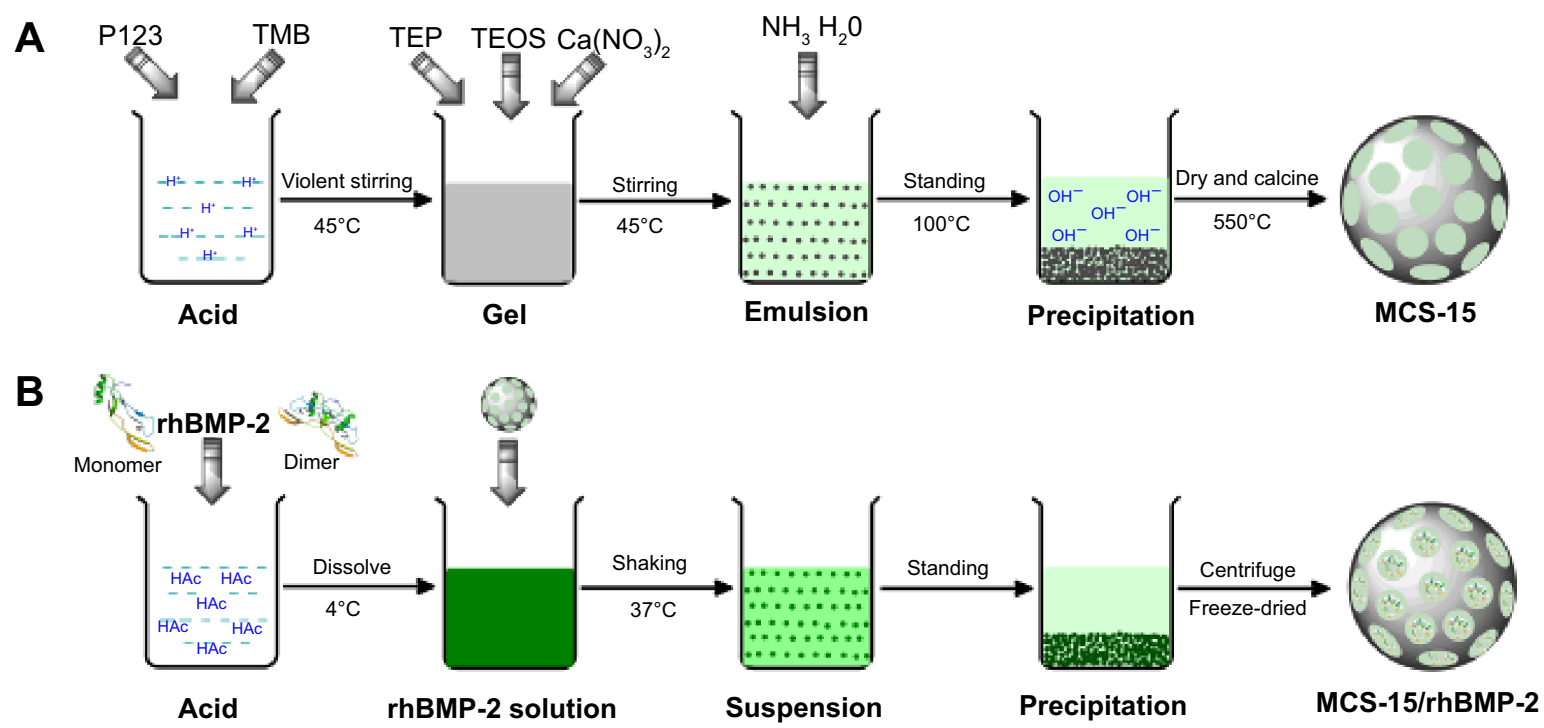

Figure I Schematic diagram showing the preparation process of (A) MCS-15 with mesoporous structure and (B) MCS-15/rhBMP-2 system. Abbreviations: MCS, mesoporous calcium-silicon; rhBMP, recombinant human bone morphogenetic protein; TMB, 3,5-mesitylene; TEP, triethyl phosphate; TEOS, ethyl silicate. 


\section{Characterization of MCS-I5}

The phase composition and microstructure of MCS-15 were determined using small-angle X-ray diffraction (Rigaku D/max 2550VB/PC; Rigaku, Tokyo, Japan) and high-resolution transmission electron microscopy (HR-TEM; JEM-1400; JEOL, Tokoyo, Japan). The pore structure parameters, including the specific surface area, pore size, pore volume, and pore size distribution, were determined using Brunauer-Emmett-Teller (BET) and Barrett-Joyner-Halenda analyses. The surface morphology of the mesoporous materials was examined using scanning electron microscopy (SEM; JSM-6360LV; JEOL).

\section{In vitro degradation of MCS-I5}

The degradation property of MCS- 15 was evaluated at $37^{\circ} \mathrm{C}$ in $0.05 \mathrm{M}$ Tris(hydroxymethyl) aminomethane- $\mathrm{HCl}$ buffer solution ( $\mathrm{pH}$ 7.4) (Tris-HCl; Sinopharm Chemical Reagent Co., Ltd.,). The MCS-15 was immersed in the buffer solution in the vials. The vials were then loaded onto an orbital shaker with the speed set at $120 \mathrm{rpm}$. At the scheduled time, the MCS-15 was separated from the solution by centrifugation, washed, and dried at $120^{\circ} \mathrm{C}$ to a constant weight. The relative weight loss percentage of the samples was calculated by:

$$
\text { Weight loss }(\%)=\left[\left(W_{0}-W_{t}\right) / W_{0}\right] \times 100 \%
$$

where $W_{0}$ and $W_{t}$ are the initial weight and the weight after a specific immersion time period $t$, respectively.

\section{Preparation of MCS-I5/rhBMP-2 system}

The rhBMP-2 solution $(0.5 \mathrm{mg} / \mathrm{mL})$ was first prepared by dissolving the lyophilized rhBMP-2 (Ruibang Biological Materials Co., Ltd., Shanghai, People's Republic of China) in $0.02 \mathrm{M}$ dilute acetic acid. The MCS-15 $(0.5 \mathrm{~g})$ was then added to $20 \mathrm{~mL}$ rhBMP-2 solution. The resulting solution was placed in a refrigerator shaker with a speed of $80 \mathrm{rpm}$ at $37^{\circ} \mathrm{C}$. The solution was centrifuged after $0,0.5,1,2,4,8$, 12,24 , and 48 hours. The supernatant $(0.5 \mathrm{~mL})$ was taken out from the vials and its absorbance was measured at $562 \mathrm{~nm}$. Phosphate-buffered saline (PBS) solution $(0.5 \mathrm{~mL})$ was added into the vials to keep the sample volume unchanged. The protein concentration was determined by comparing with a standard curve. The adsorption capacity, ie, the weight ratio of the adsorbed proteins onto the materials, was calculated subsequently. The MCS-15 (MCS-4 as a control) loaded with rhBMP-2 was then precipitated by centrifugation, dried at $37^{\circ} \mathrm{C}$, and preserved in a sealed container at $4^{\circ} \mathrm{C}$. The schematic diagram of the preparation procedure is shown in Figure 1B.

\section{RhBMP-2 release from MCS- I5}

The MCS-15/rhBMP-2 system (MCS-4/rhBMP-2 system as a control) was first placed in a centrifuge tube $(10 \mathrm{~mL})$, and simultaneously, $3 \mathrm{~mL}$ of PBS buffer solution $(\mathrm{NaCl}$ $137 \mathrm{mM}, \mathrm{KCl} 2.7 \mathrm{mM}, \mathrm{Na}_{2} \mathrm{HPO}_{4} \cdot 12 \mathrm{H}_{2} \mathrm{O} 8 \mathrm{mM}, \mathrm{KH}_{2} \mathrm{PO}_{4}$ $1.5 \mathrm{mM}, \mathrm{pH} 7.4$ ) was added. The leading suspension was incubated in a refrigerator shaker with a speed of $80 \mathrm{rpm}$ at $37^{\circ} \mathrm{C}$. The supernatant was then removed from the samples and the rhBMP-2 quantity in the supernatant was determined using the Bradford protein assay. The released quantity of rhBMP-2 was measured at $0,0.25,0.5,1,2,4,8,12,24,48$, $72,120,200,240,300$, and 360 hours. The release curve was constructed by plotting the release percentage of rhBMP-2 (the ratio of released amount to the total rhBMP-2 amount) against the time.

\section{Cell viability and morphology}

The MTT (3-(4,5-Dimethylthiazol-2-yl)-2,5-diphenyltetrazolium bromide) assay was employed to evaluate the influences of MCS-15 loading rhBMP-2 on cell viability (MCS-4 loading rhBMP-2, MCS-15, and MCS-4 as controls) through the mitochondrial activity measurement. The evaluation was conducted by culturing hMSCs (purchased from the Chinese Academy of Sciences, Shanghai, People's Republic of China) on the samples in 96-welled plates for 1,3 , and 5 days. The hMSCs were seeded in 96-welled plates by transferring a certain amount of the cell suspension into each well. The samples were subsequently added into the wells at a concentration of $1 \mathrm{mg} / \mathrm{mL}$ and cultured with the cells at $37^{\circ} \mathrm{C}$ in a $5 \% \mathrm{CO}_{2}$ incubator for 1,3 , and 5 days. About $4 \mathrm{~mL}$ MTT (Amresco, Solon, OH, USA) solution $(5 \mathrm{mg} / \mathrm{mL}$ ) was added onto each plate and incubated at $37^{\circ} \mathrm{C}$ for 4 hours. The culture medium was sucked out, and the solid residues were washed twice using $150 \mu \mathrm{L}$ PBS. Thereafter, PBS was replaced with an equal amount $(150 \mu \mathrm{L})$ of dimethyl sulfoxide (DMSO, Sigma, St Louis, MO, USA). The plates were vibrated for 15 minutes to dissolve the formazan. Finally, the optical density (OD) was measured at $490 \mathrm{~nm}$ using an ELX Ultra Microplate Reader (Bio-tek, Winooski, VT, USA).

The morphology of the hMSCs was examined by visualizing the filamentous actin of the cytoskeleton using confocal laser scanning microscopy (Leica TCS SP2; Leica Microsystems, Heidelberg, Germany). Prior to the microscopic examination, the samples were pressed into disks $(\Phi, 12 \times 2 \mathrm{~mm})$ and rhBMP-2 solution $(40 \mu \mathrm{L})$ with a concentration of $0.5 \mathrm{mg} / \mathrm{mL}$ was added dropwise to the specimens. The samples were then put into 24 wells, and the hMSCs were seeded on the samples at a density of $5.0 \times 10^{4}$ cells per 
well. According to the protocol, the cells were fixed with $2.5 \%$ glutaraldehyde for 15 minutes and permeabilized with $0.1 \%$ Triton X-100 in PBS for another 15 minutes. After washing with PBS three times, the cells were incubated with fluorescein isothiocyanate (FITC)-Phalloidin (Abcam, Sigma-Aldrich Co.) for 1 hour and then counterstained with DAPI (4',6-diamidino-2-phenylindole) (Molecular Probe, Sigma-Aldrich Co.) to observe the nucleus after 10 minutes. The cell morphologies were visualized using confocal laser scanning microscopy.

\section{Alkaline phosphatase activity}

The alkaline phosphatase (ALP) activity was measured by the ALP assay in osteogenic medium at 4 and 7 days. Briefly, $2.5 \times 10^{4}$ of hMSCs were seeded on the MCS-15, MCS-15/ rhBMP-2, MCS-4, and MCS-4/rhBMP-2 samples, which were placed in 24 -welled plates, and then cultured at $37^{\circ} \mathrm{C}$ and $100 \%$ humid atmosphere with $5 \% \mathrm{CO}_{2}$. At each culture period, the culture medium was removed from the samples and the cells were washed with PBS. About $1 \mathrm{~mL}$ of $0.2 \%$ Nonidet P-40 solution (NP-40) was added to each well at room temperature for substrate solution (1 mg/mL, $\mathrm{pH} 9$ ) composed of $0.1 \mathrm{~mol} / \mathrm{L}$ glycine and $0.5 \mathrm{mmol} / \mathrm{L} \mathrm{MgCl}_{2}$ in $1 \mathrm{M}$ diethanolamine buffer, and incubated at $37^{\circ} \mathrm{C}$ for 15 minutes. Sodium hydroxide $(100 \mu \mathrm{L}, 0.1 \mathrm{M})$ was added to stop the reaction. Then the OD was quantified with a microplate reader (SPECTRAmax 384, Molecular Devices, Sunnyvale, CA, USA) at the wavelength of $405 \mathrm{~nm}$. The ALP activity was expressed as OD value per total protein. The total protein content was determined using the bicinchoninic acid protein assay kits and a series of bovine serum albumin standards.

\section{A}

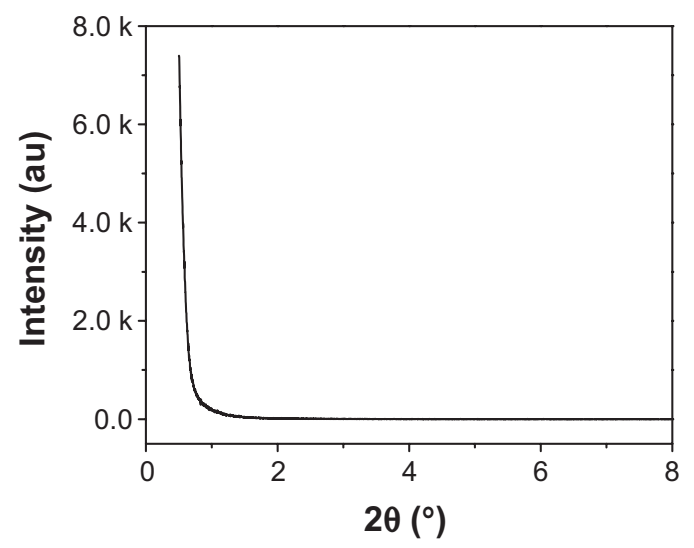

\section{Statistical analysis}

All quantitative data were analyzed with Origin 8.0 (OriginLab Corporation, Northampton, MA, USA) and expressed as the mean \pm standard deviation $(M \pm S D)$. Statistical comparisons were carried out using one-way analysis of variance (ANOVA) with post hoc test.

\section{Results}

\section{X-ray diffraction of MCS-I 5}

The typical small-angle X-ray diffraction profiles of the MCS-15 and MCS-4 are shown in Figure 2A and B. The diffraction peaks of MCS-15 disappeared (Figure 2A), suggesting that the typical mesoporous structure was destroyed by the pore-expanding agents. In contrast, the MCS-4 exhibited a well-resolved (100) peak and very weak $(110,200)$ signals (Figure 2B), indicating a high degree of hexagonal mesoscopic organization (p6mm). The wide-angle X-ray diffraction spectra of the MCS-15 and MCS-4 are shown in Figure $3 \mathrm{~A}$ and $\mathrm{B}$, in which the MCS-15 exhibited a single broad X-ray diffraction peak $\left(2 \theta=10^{\circ}-40^{\circ}\right)$ and no obvious difference between MCS-15 and MCS-4 spectra was identified. In addition, the energy dispersive spectrometer of MCS-15 is shown in Figure 3C; it was found that there were $\mathrm{Si}$ and $\mathrm{Ca}$ elements in the MCS-15.

\section{BET analysis}

The $\mathrm{N}_{2}$ adsorption-desorption isotherms and pore diameter distribution of the MC1-15 and MCS-4 are shown in Figure 4A and B and Figure 5A and B, respectively, which are very different, indicating the distinctive pore structures. In the isotherm of MCS-15 that was synthesized using a poreexpanding agent, a sudden jump in the amount of adsorbed

B

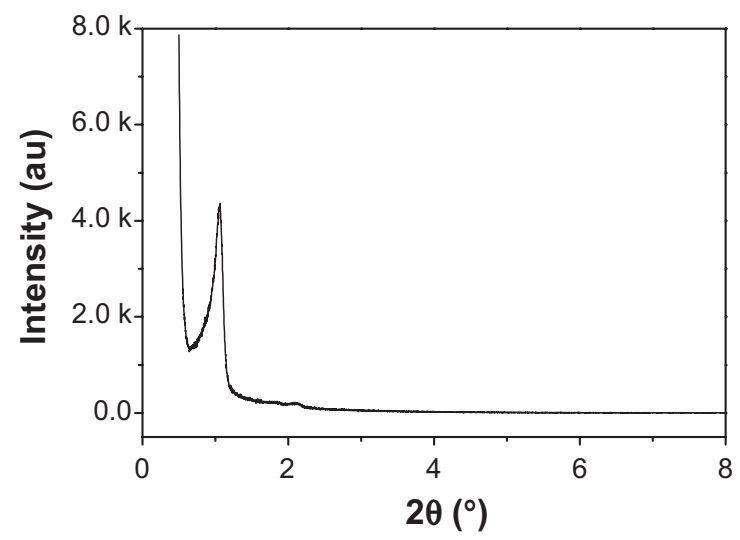

Figure 2 Small-angle X-ray patterns of MCS-15 (A) and MCS-4 (B). Abbreviations: au, arbitrary units; MCS, mesoporous calcium-silicon. 

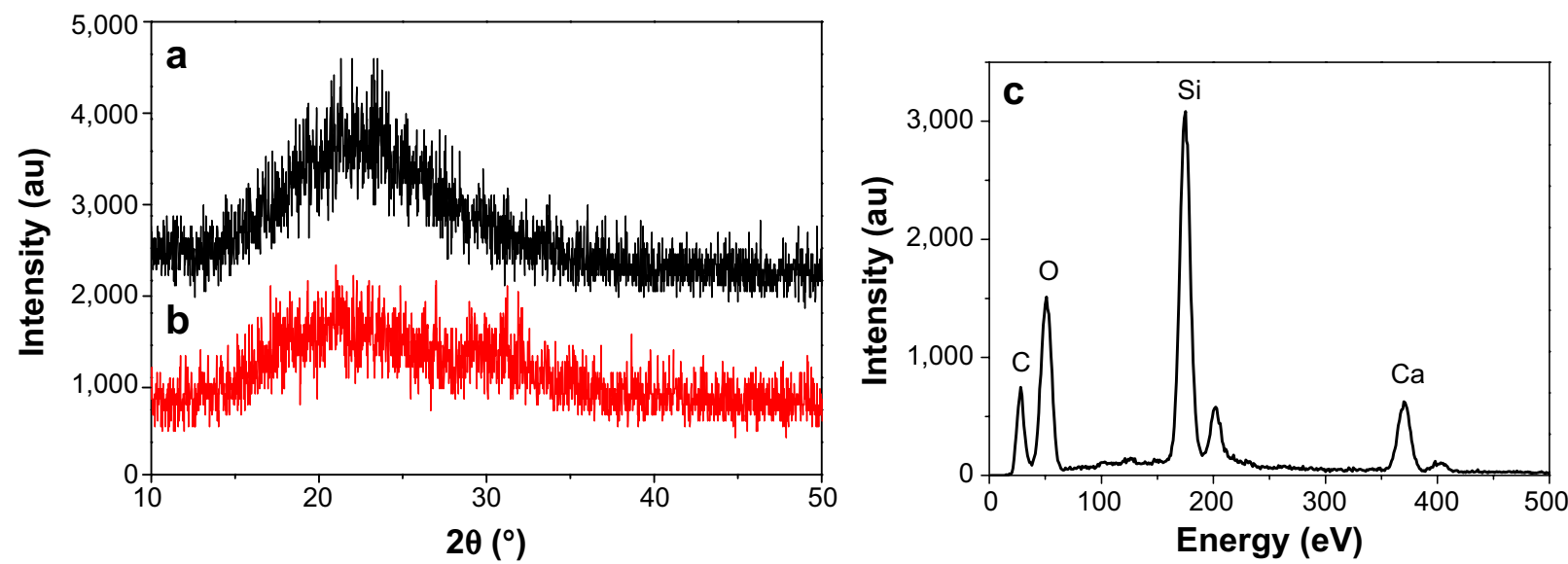

Figure 3 Wide-angle X-ray diffraction of MCS-I5 (a) and MCS-4 (b), and EDS of MCS-I5 (c).

Abbreviations: au, arbitrary units; EDS, energy dispersive spectrometer; MCS, mesoporous calcium-silicon.

$\mathrm{N}_{2}$ within a $\mathrm{P} / \mathrm{P}_{0}$ range from 0.8 to 1.0 was found. The $\mathrm{BET}$ specific surface area, pore size, and pore volume of MCS-15 were $257.8 \mathrm{~m}^{2} / \mathrm{g}, 15 \mathrm{~nm}$, and $1.43 \mathrm{~cm}^{3} / \mathrm{g}$, respectively. However, the isotherm of MCS-4 was a typical type IV isotherm, in which the hysteresis loop was clearly evident, implying a highly consistent pore structure. The BET surface area, pore size, and pore volume of MCS-4 were $485.6 \mathrm{~m}^{2} / \mathrm{g}$, $4 \mathrm{~nm}$, and $0.48 \mathrm{~cm}^{3} / \mathrm{g}$, respectively, considerably different from those of MCS-15.

\section{TEM and SEM examinations}

The morphology and microstructure of the MCS-15 examined by TEM are shown in Figure 6 . The MCS-15 synthesized using TMB showed a disordered mesoporous structure, as well as pretty thick pore walls and larger pore size $(15 \mathrm{~nm})$, whereas MCS-4 synthesized without using TMB possessed a highly ordered pore structure with a smaller pore size and thicker pore walls. The SEM images of the surface morphology of the MCS-15 are shown in Figure 7. The MCS-15 was made of irregular particles with an interconnected porous structure, while the MCS-4 was made of regular short rodlike particles without obvious pores on the surface of the particles.

\section{Degradability of MCS- 5 in Tris- $\mathrm{HCl}$ solution}

The in vitro degradation property of MCS-15 was determined by testing the weight loss ratio of the samples after soaking in Tris- $\mathrm{HCl}$ solution for different time periods. The weight loss of both MCS-15 and MCS-4 is shown in Figure 8. It was found that the weight loss increased with the immersion time for both the materials, and the weight loss of MCS-15 and MCS-4 were $50 \mathrm{wt} \%$ and $30 \mathrm{wt} \%$, respectively, after soaking for 56 days. The weight loss of MCS-15 was significantly higher than that of MCS-4,
A

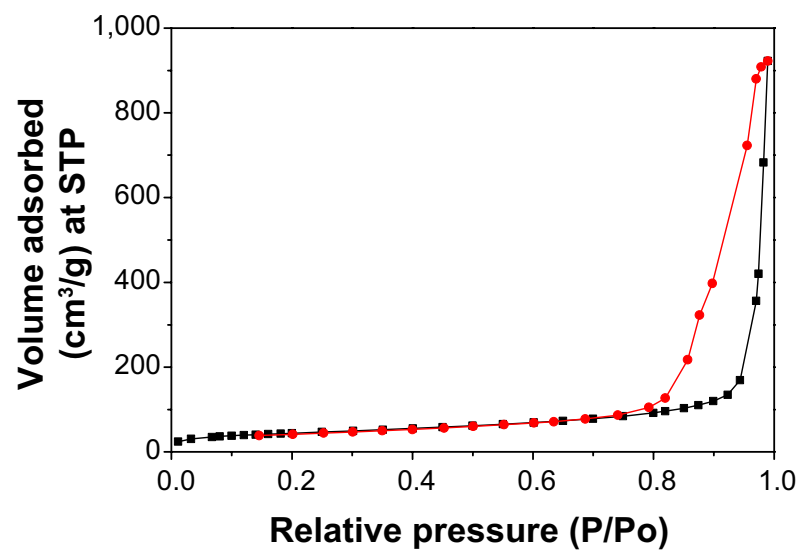

B

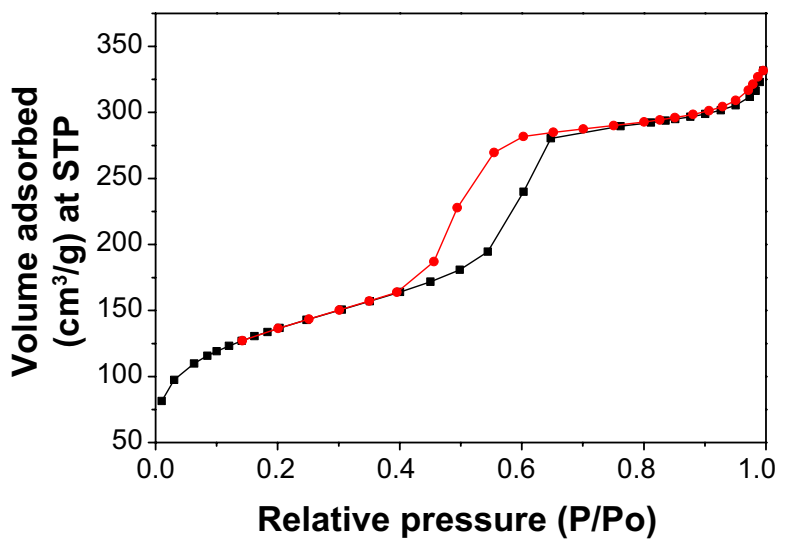

Figure $4 \mathrm{~N}_{2}$ adsorption-desorption isotherms of the samples: MCS-I5 (A) and MCS-4 (B). Abbreviations: MCS, mesoporous calcium-silicon; STP, standard temperature and pressure. 

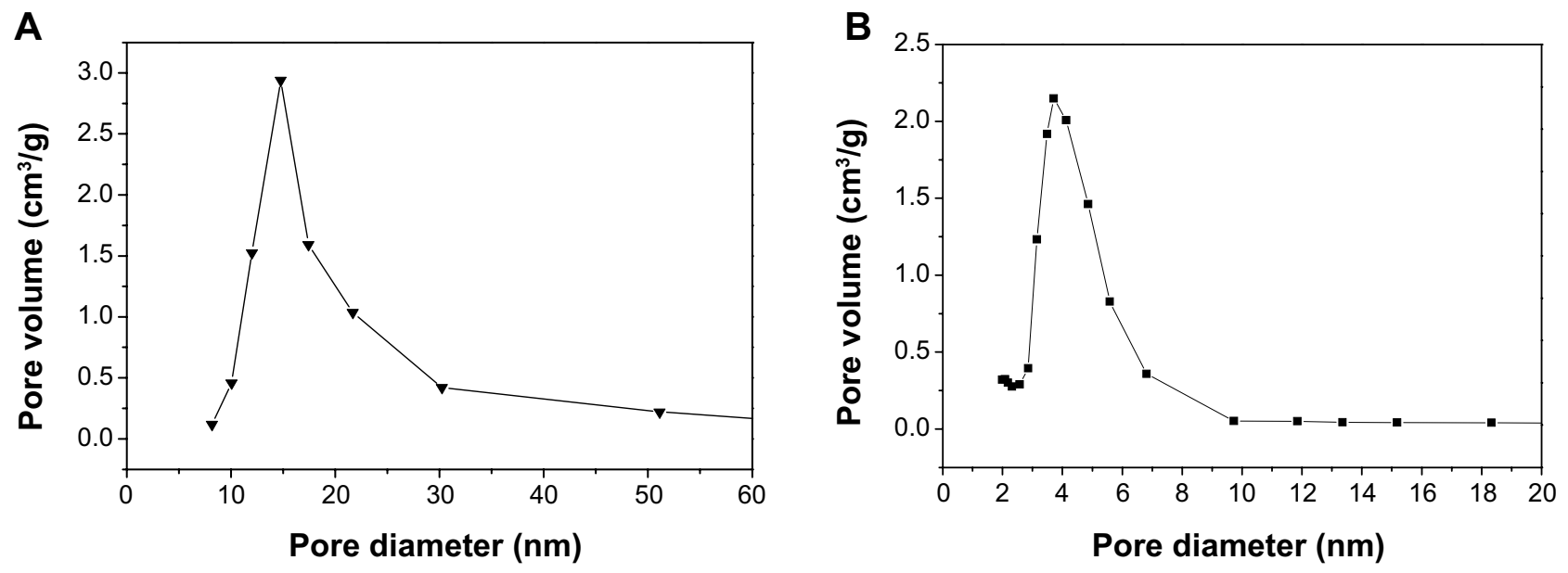

Figure 5 Pore diameter distribution of the samples MCS-15 (A) and MCS-4 (B) Abbreviation: MCS, mesoporous calcium-silicon.
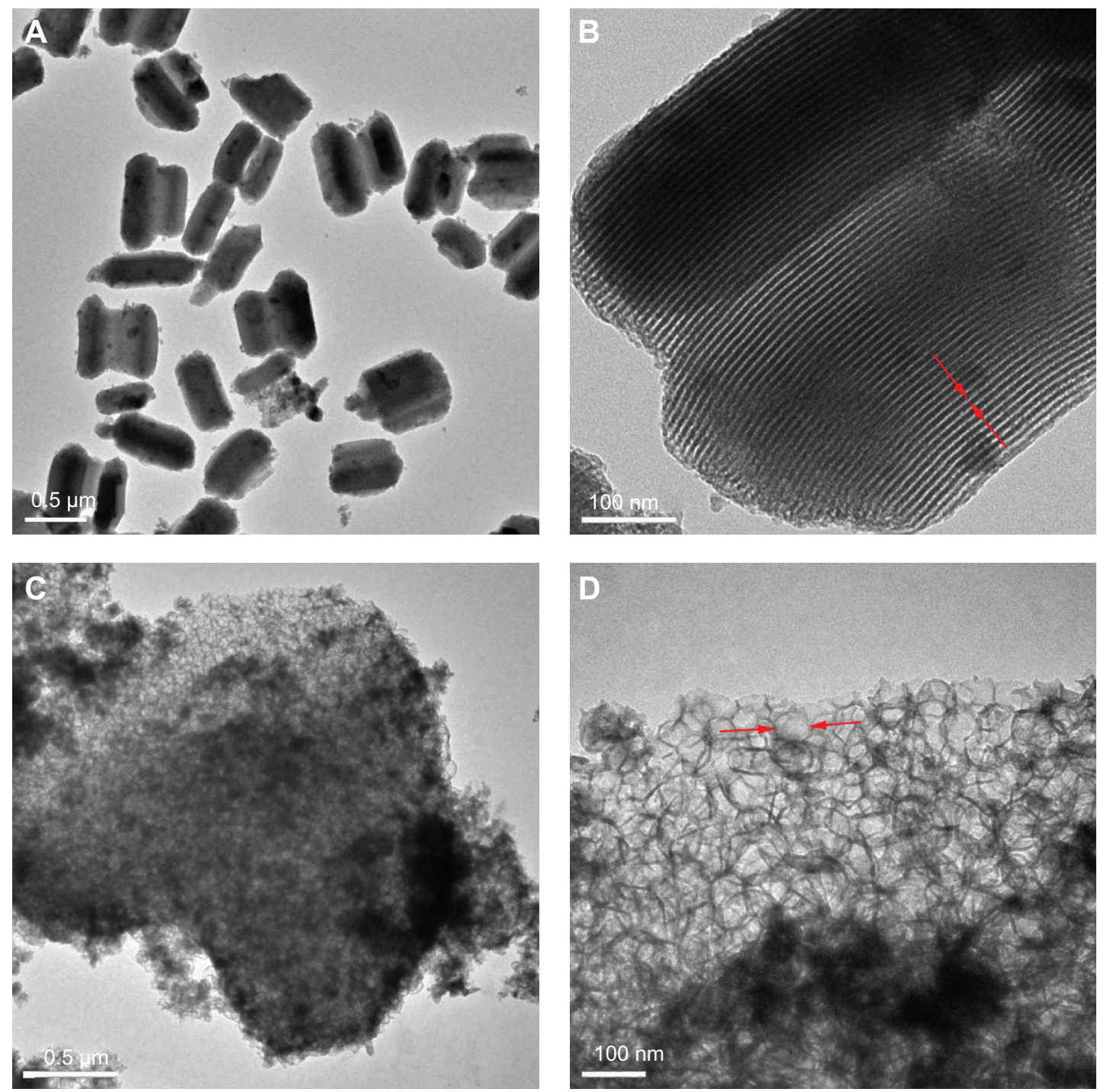

Figure 6 TEM images of the samples: (A) MCS-15, (B) magnified local area in (A), (C) MCS-4, and (D) high-resolution TEM image of MCS-4. Note: Arrows indicate the mesoporous structure and pore size.

Abbreviations: MCS, mesoporous calcium-silicon; TEM, transmission electron microscope. 

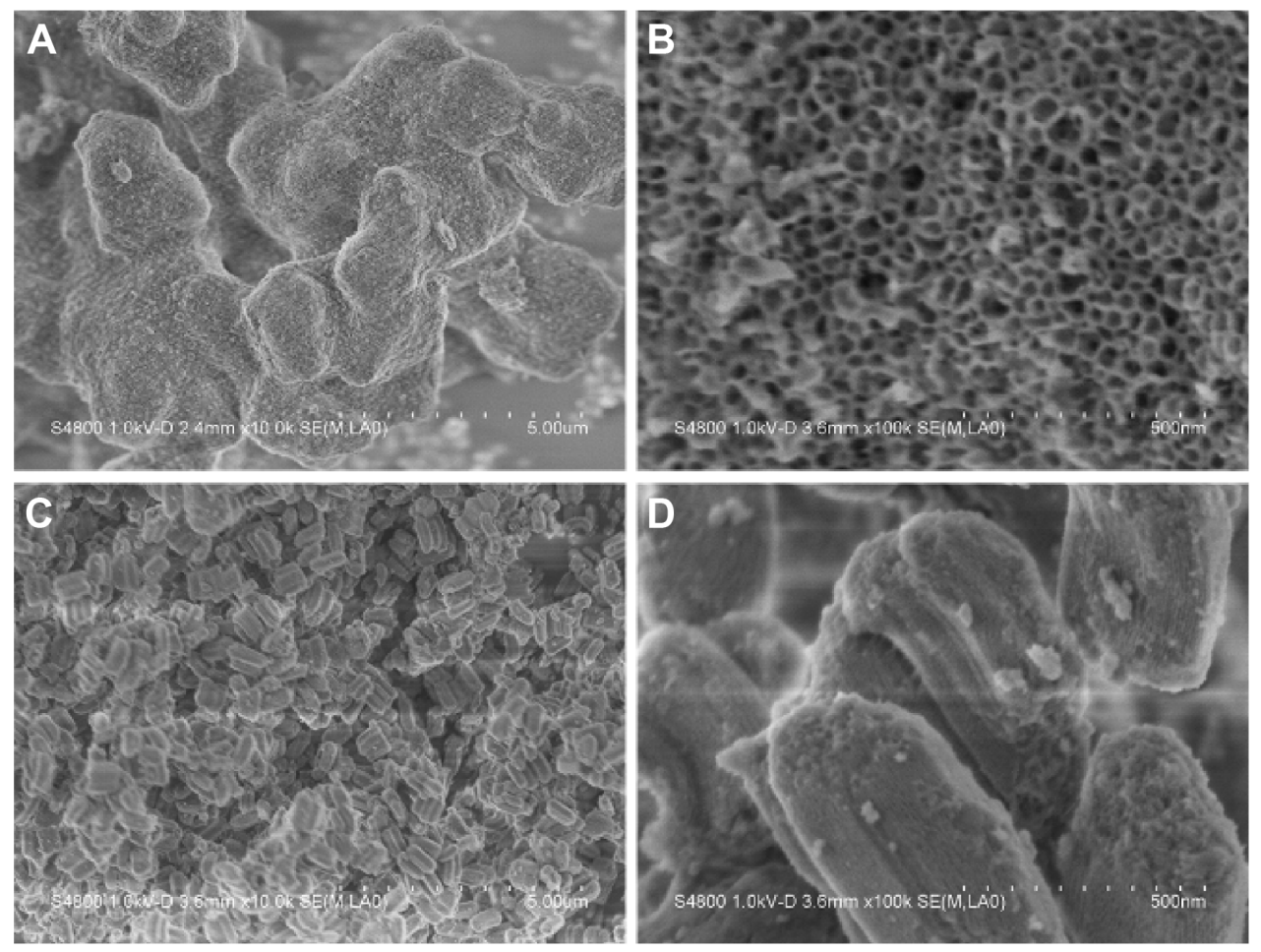

Figure 7 SEM images of the sample.

Notes: (A) MCS-15, (B) magnified local area in (A), (C) MCS-4, and (D) magnified local area in (C).

Abbreviations: MCS, mesoporous calcium-silicon; SEM, scanning electron microscope.

indicating that the degradation rate of MCS-15 was faster than that of MCS-4.

\section{MCS-I 5 loading and release of rhBMP-2}

Figure 9A shows the profile of rhBMP-2 loaded onto MCS-15 and MCS-4 after immersion in PBS solution for different times. After 24 hours, the amount of rhBMP-2 adsorbed onto MCS-15 was $45 \mathrm{mg} / \mathrm{g}$ (per unit gram), which was more

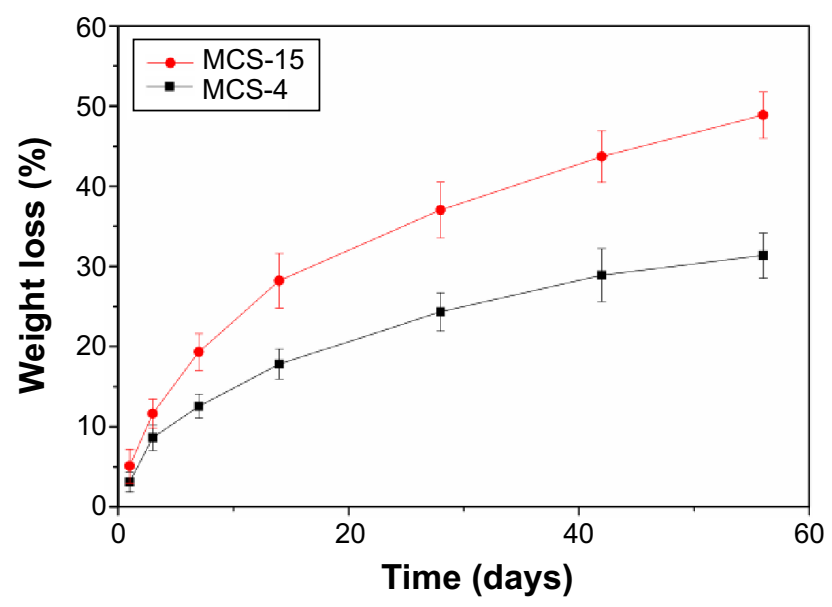

Figure 8 Weight loss of MCS- 15 and MCS-4 immersed in Tris- $\mathrm{HCl}$ solution with time. Abbreviation: MCS, mesoporous calcium-silicon. than twice that adsorbed onto MCS-4 $(20 \mathrm{mg} / \mathrm{g})$. The results indicated that MCS-15 had higher capacity to encapsulate large amounts of rhBMP-2.

Figure 9B shows the profile of rhBMP-2 released from MCS-15 and MCS-4 after immersion in PBS solution for different times. Even after 14 days, 75 wt $\%$ of rhBMP-2 was found to be released from MCS-15, while more than $80 \mathrm{wt} \%$ of rhBMP- 2 was found to be released from MCS-4 within 3 days, showing a burst release of rhBMP-2. The results indicated that MCS-15 exhibited a sustained-release behavior as compared with MCS-4.

\section{Cell proliferation and morphology}

Proliferation of hMSCs cultured on both the MCS-15/ rhBMP-2 and MCS-4/rhBMP-2 systems was assessed through the mitochondrial activity measurement, using the MTT assay. Figure 10 reveals that the OD values for all the samples increased with time, and the OD values for both MCS-15/rhBMP-2 and MCS-4/rhBMP-2 systems were significantly higher than those for MCS-15 and MCS-4 without rhBMP-2 at 1,3 , and 5 days $(P<0.05)$. In addition, the OD value for MCS-4/rhBMP-2 was higher than that for MCS-15/rhBMP-2 system at 1 and 3 days because of more rhBMP-2 release from MCS-4/rhBMP-2 (initial burst 
A

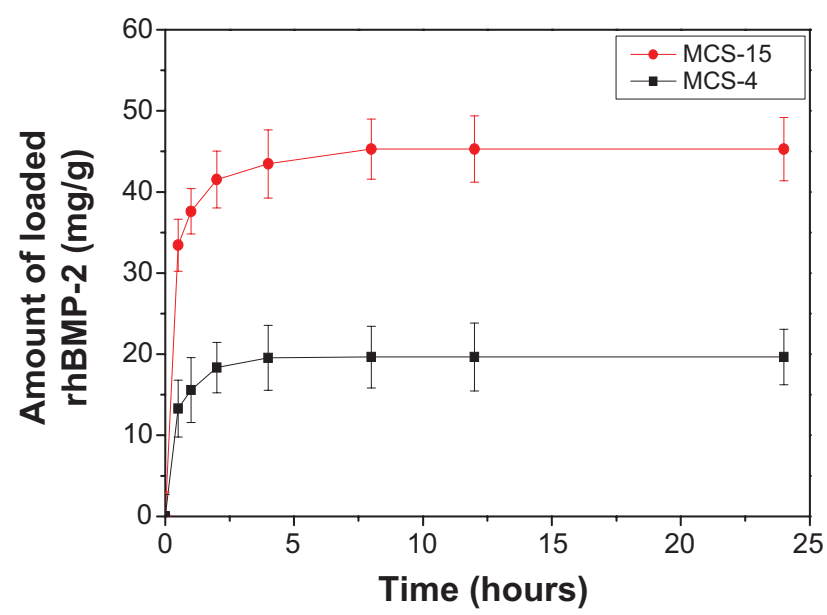

B

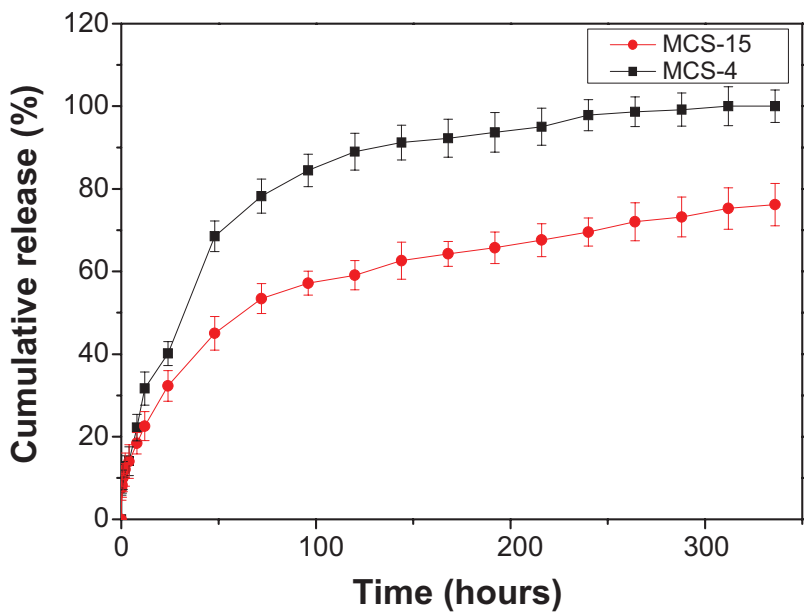

Figure 9 Profile of rhBMP-2 loaded on MCS-15 (A) and released (B) from MCS-15 (MCS-4 as a control).

Abbreviations: MCS, mesoporous calcium-silicon; rhBMP, recombinant human bone morphogenetic protein.

release from MCS-4). However, the OD value for MCS-15/ rhBMP-2 system was significantly higher than that for MCS-4/rhBMP-2 at 5 days $(P<0.05)$. The results indicated that MCS-15/rhBMP-2 system with sustained release of rhBMP-2 facilitated continued cell proliferation.

The cytoskeletal morphologies of the hMSCs cultured on both the MCS-15/rhBMP-2 and MCS-4/rhBMP-2 systems are shown in Figure 11. It was found that the spindle cells extended and spread well on both the MCS-15/rhBMP-2 and MCS-4/rhBMP-2 systems. However, the number of cells on MCS-15/rhBMP-2 was significantly higher than that on MCS-4/rhBMP- 2 at 5 days $(P<0.05)$. In addition, the number

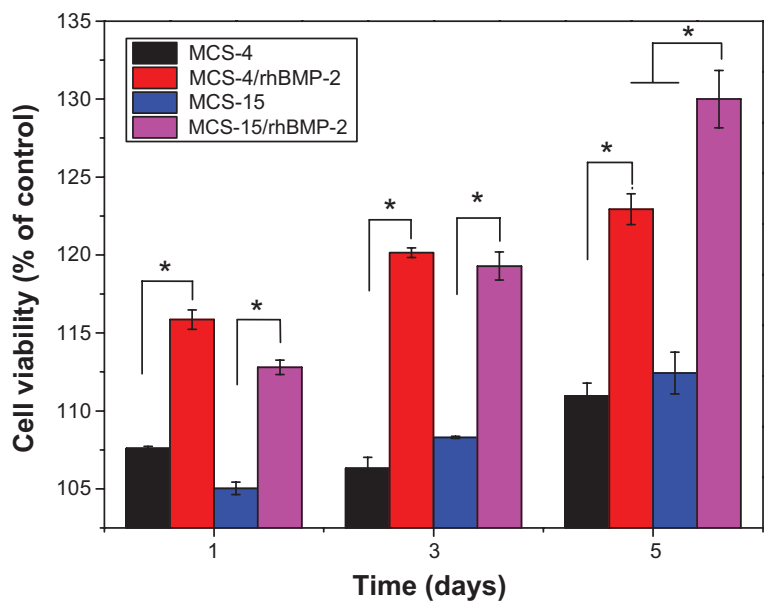

Figure 10 Proliferation of hMSCs cultured on both MCS-15/rhBMP-2 and MCS-4/ rhBMP-2 at I, 3, and 5 days through the mitochondrial activity measurement (MCS-15 and MCS-4 without loaded rhBMP-2 as controls).

Note: $* P<0.05$.

Abbreviations: MCS, mesoporous calcium-silicon; rhBMP, recombinant human bone morphogenetic protein; hMSCs, human mesenchymal stem cells. of cells on both the MCS-15/rhBMP-2 and MCS-4/rhBMP-2 systems was significantly higher than that on MCS-15 and MCS-4 without rhBMP-2 at 5 days $(P<0.05)$. The results indicated that the MCS-15/rhBMP-2 system exhibited significant effects on hMSC viability among all the groups.

\section{Alkaline phosphatase activity}

ALP activity of hMSCs cultured on MCS-15/rhBMP-2 and MCS-4/rhBMP-2 systems was assessed at 4 and 7 days. Figure 12 reveals that the ALP activities for both MCS-15/ rhBMP-2 and MCS-4/rhBMP-2 systems were significantly higher than those for MCS-15 and MCS-4 without rhBMP-2 at 4 and 7 days $(P<0.05)$. At 4 days, ALP expressed at lower levels, and no significant differences were detected for both MCS-15/rhBMP-2 and MCS-4/rhBMP-2 systems. However, the ALP activity of hMSCs on MCS-15/rhBMP-2 system was significantly greater than that on MCS-4/rhBMP-2 at 7 days $(P<0.05)$.

\section{Discussion}

Over the past few years, mesoporous materials have shown the potential application as drug-release carriers because of their stable mesoporous structures, tunable pore size, and high pore volume, allowing sufficient incorporation of large quantities of molecules within the nanoporous structures. ${ }^{3}$ A study has shown that the pore size and pore volume were two key factors for loading capacity of the drug molecules and subsequent release profiles. ${ }^{15}$ Our previous study indicated that MCS-4 with the pore size of $4 \mathrm{~nm}$ was too small to load more biomacromolecules of rhBMP-2 and that it not only had a 

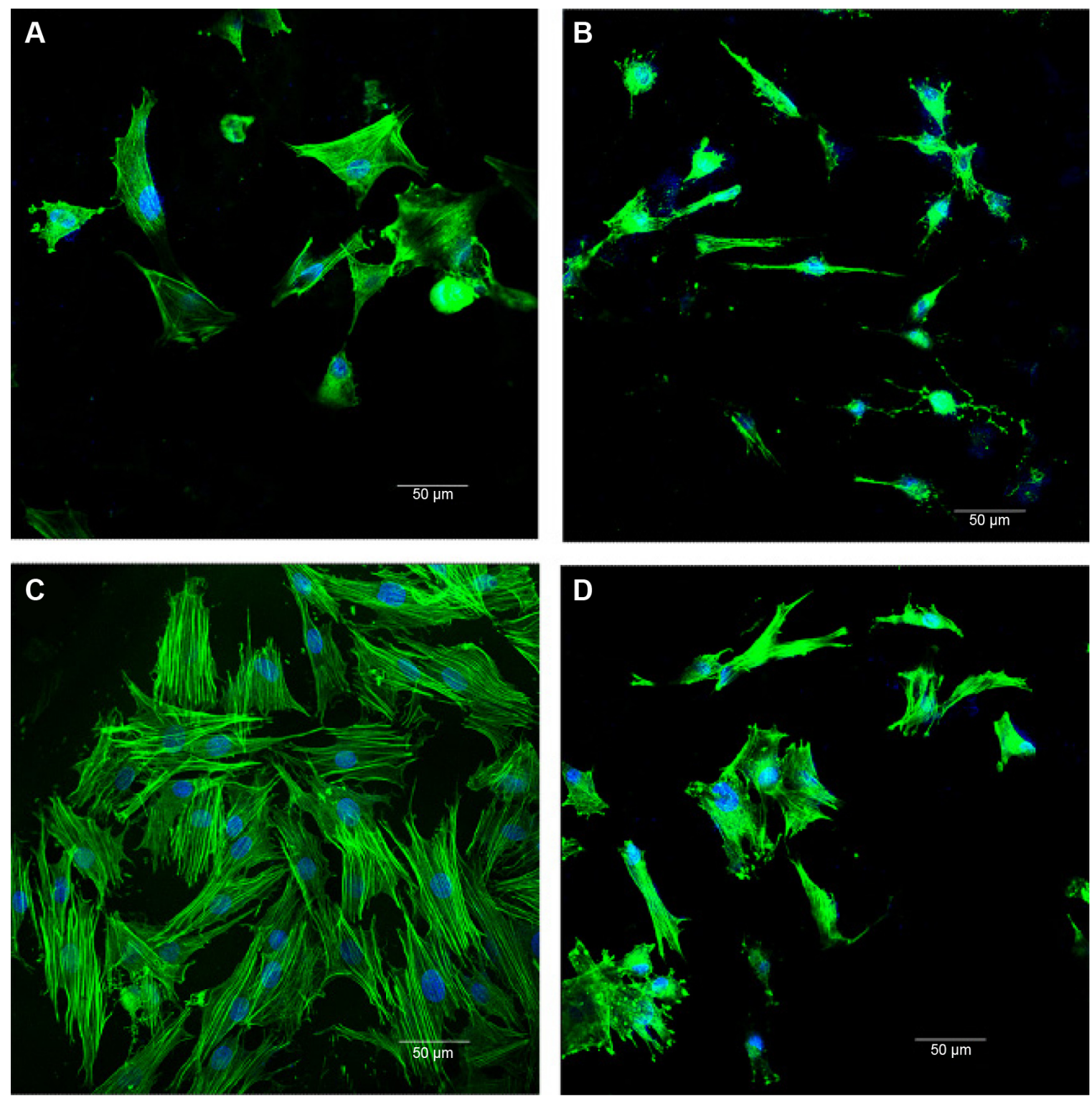

Figure I I Cytoskeletal morphology and spreading of the hMSCs on the samples for 5 days, (A) MCS-I5, (B) MCS-4, (C) MCS-15/rhBMP-2, and (D) MCS-4/rhBMP-2. Abbreviations: hMSCs, human mesenchymal stem cells; MCS, mesoporous calcium-silicon.

lower loading capacity but also resulted in an undesirable release behaviour, such as an initial burst release of rhBMP-2. Therefore, in the current study we designed the MCS- 15 with larger pore size and higher pore volume for the delivery of rhBMP-2. The results showed that the pore size and pore volume of MCS- 15 were $15 \mathrm{~nm}$ and $1.43 \mathrm{~cm}^{3} / \mathrm{g}$, while those of MCS-4 were $4 \mathrm{~nm}$ and $0.48 \mathrm{~cm}^{3} / \mathrm{g}$, respectively.

As for bone tissue regeneration, it is expected that the biomaterials could be degradable and gradually replaced by new bone tissue when implanted in vivo. ${ }^{16}$ In this study, the MCS-15 was found to be degradable in Tris- $\mathrm{HCl}$ solution; the weight loss of MCS- 15 was $50 \mathrm{wt} \%$ and that of MCS-4 was $30 \mathrm{wt} \%$ after soaking in Tris- $\mathrm{HCl}$ for 56 days (shown in Figure 8). The faster degradation rate of MCS-15 was due to its larger pore size and higher pore volume in comparison with MCS-4. The results indicated that increasing the pore size and pore volume of MCS-15 could enhance the degradability. Therefore, it can be suggested that change in the microstructure of the implanted biomaterials could modulate their degradability in vivo.

The characteristics of mesoporous materials such as tunable pore size and high pore volume make it possible to adsorb proteins and drug molecules highly efficiently and release them from the mesostructured matrices in a sustained manner. ${ }^{17,18}$ The biomacromolecule BMP is the most effective osteoinduction factor and displays potent osteogenesis 


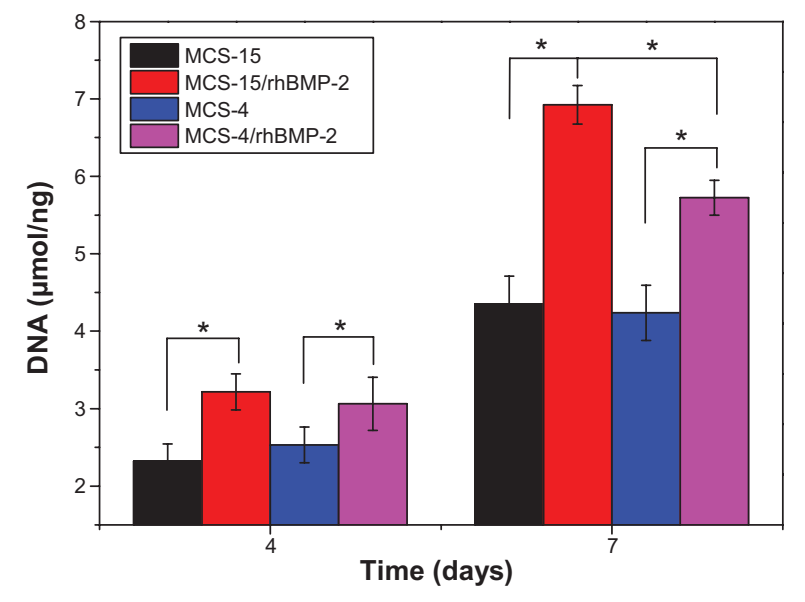

Figure 12 ALP activity of hMSCs cultured on MCS- I5/rhBMP-2 and MCS-4/rhBMP-2 at 4 and 7 days (MCS-I5 and MCS-4 without loaded rhBMP-2 as controls). Note: $* p<0.05$.

Abbreviations: ALP, alkaline phosphatase; DNA, deoxyribonucleic acid; hMSCs, human mesenchymal stem cells; MCS, mesoporous calcium-silicon; rhBMP, recombinant human bone morphogenetic protein.

activity both in vitro and in vivo. In this study, the results showed that the amount of rhBMP-2 adsorbed onto the MCS-15 was $45 \mathrm{mg} / \mathrm{g}$, more than twice that adsorbed onto MCS-4 (20 mg/g) (shown in Figure 9). Obviously, MCS-15, with a pore size of $15 \mathrm{~nm}$ and pore volume of $1.43 \mathrm{~cm}^{3} / \mathrm{g}$, was able to absorb a large amount of rhBMP-2 macromolecules, compared with MCS-4.

The size of rhBMP- 2 macromolecule $(7 \times 3.5 \times 2.5 \mathrm{~nm})$ was smaller than the pore size of MCS-15 (around $15 \mathrm{~nm}$ ), and hence rhBMP-2 would be absorbed not only on the external surface but also on the inside of the MCS-15 (mesopores). In addition, the higher pore volume of MCS-15 $\left(1.43 \mathrm{~cm}^{3} / \mathrm{g}\right)$ could load more rhBMP-2 onto the internal surface of the mesoporous materials. However, the MCS-4 with the smaller pore size of $4 \mathrm{~nm}$ and lower pore volume of $0.48 \mathrm{~cm}^{3} / \mathrm{g}$ absorbs only a small amount of rhBMP-2 (which might be only absorbed on the external surface of the mesoporous materials). The results indicated that the larger pore size and higher pore volume of MCS-15 were favorable for rhBMP-2 adsorption, thus making MCS-15 a promising carrier for loading bioactive macromolecules.

The low loading capacity of biomaterials for biomacromolecules is due not only to the pore size but also to the pore volume, which make macromolecules unable to enter into the smaller mesopores. ${ }^{19}$ Furthermore, the loaded macromolecules released completely from the biomaterials over a very short time, showing an initial burst release behavior. ${ }^{20}$ The rhBMP-2 release experiments in this study showed that $75 \mathrm{wt} \%$ of rhBMP-2 was released from MCS-15 even after 14 days, obviously indicating sustained release of rhBMP-2 from MSC-15. However, more than $80 \mathrm{wt} \%$ of rhBMP-2 was released from MCS-4 within 3 days, indicating that the release of rhBMP-2 from MSC-4 was very quick, showing an initial burst release. In addition, it was found that the rhBMP-2 was released from MCS-4 almost immediately because of the small amount of rhBMP-2 adsorbed on the external surface of the MCS-4 (material surface). As a result, the MCS-4 could not achieve the long-term sustained release of rhBMP-2. Importantly, a large amount of rhBMP-2 could be loaded onto the MCS-15 by adsorption processes, which could avoid initial burst release of rhBMP-2. Therefore, the MCS-15 was a very promising carrier for sustained release of rhBMP-2.

The results of cell culture experiments showed that the proliferation of hMSCs on both the MCS-15/rhBMP-2 and MCS-4/rhBMP-2 systems was significantly higher than that on MCS-15 and MCS-4 without loading rhBMP-2, as shown in Figure 10, indicating that rhBMP-2 loaded MCS could promote cell proliferation because rhBMP-2 is a growth factor, and its bioactivity has been proved to facilitate cell proliferation and differentiation. ${ }^{21}$ In addition, the proliferation of hMSCs on MCS-4/rhBMP-2 was higher than that on MCS-15/rhBMP-2 system at 1 and 3 days because of more rhBMP-2 release from MCS-4/rhBMP-2 (initial burst release, rhBMP-2 absorbed on the external surface of the materials) to stimulate cell growth. However, the cell proliferation on MCS-15/rhBMP-2 system was significantly higher than that on MCS4/rhBMP-2 at 5 days, indicating that the MCS-15 could release rhBMP-2 sustainably and persistently promote cell proliferation. The slow release of rhBMP-2 from MCS-15 might be due to the retention of a large amount of rhBMP-2 by the internal mesopores and thus preservation of its bioactivity.

The hMSCs showed a spindle shape, as shown in Figure 11, which could extend and spread well when cultured on the samples of MCS-15/rhBMP-2, MCS-4/rhBMP-2, MCS-15, and MCS-4, indicating that all the samples had good cytocompatibility. Moreover, the number of cells on MCS-15/rhBMP-2 system was significantly higher than those on the other 3 samples at 5 days, which could be ascribed to the slow release of rhBMP-2 from MCS-15, effectively preserving the bioactivity of rhBMP-2. The results suggested that the slow release of rhBMP-2 from MCS-15 exhibited a long-lasting promoting effect on cell proliferation.

It is known that hMSCs can be differentiated into osteoblasts, and the osteoblastic differentiation capacity could be further enhanced by the osteoinductive factor of rhBMP-2. ${ }^{22}$ ALP activity has been used as an early marker 
for functionality and differentiation of osteoblasts during in vitro experiments. ${ }^{23}$ In this study, ALP activity of hMSCs on the MCS-15/rhBMP-2 was significantly higher than that on MCS-4/rhBMP-2 system at 7 days, as shown in Figure 12, indicating that the cells differentiated more quickly after being cultured on the MCS-15/rhBMP-2 system than on the MCS-4/rhBMP-2 system. This increased ALP activity probably resulted from a larger quantity of rhBMP-2 released in a sustainable manner from MCS-15 as compared with MCS-4. Our results showed that the mesoporous structures of MCS-15 with larger pore size and higher pore volume offered the possibility of adsorbing or entrapping a large amount of rhBMP-2 within the mesopores (also on the external surface of materials). Such materials improved diffusion of the guest macromolecules into the mesoporous structures. However, the MCS- 4 with the pore size of $4 \mathrm{~nm}$ could adsorb rhBMP-2 only on its external surface.

In short, the MCS-15 with the larger mesopore size of $15 \mathrm{~nm}$ and pore volume of $1.43 \mathrm{~cm}^{3} / \mathrm{g}$ can be used not only as a carrier of cells but also as a high-capacity carrier for bioactive macromolecules. Therefore, it is expected that the bone repair materials made by MCS-15 could act either as scaffolds for bone tissue regeneration or as bioactive factor carriers possessing the sustained-release ability.

\section{Conclusion}

Mesoporous calcium-silicon xerogels with the pore size of $15 \mathrm{~nm}$ and pore volume of $1.43 \mathrm{~cm}^{3} / \mathrm{g}$ were synthesized using TMB as the pore-expanding agent. The MCS-15 with the larger mesopore size and higher pore volume exhibited better degradation in Tris-HCl solution than did MCS-4 with the pore size of $4 \mathrm{~nm}$ and pore volume of $0.48 \mathrm{~cm}^{3} / \mathrm{g}$. The MCS-15 with the larger mesopore size and higher pore volume allowed sufficient incorporation of large quantities of rhBMP-2 biomacromolecules within the nanopore structure by adsorption processes, and sustained release of rhBMP-2. However, the MCS-4 with the smaller pore size and lower pore volume absorbed only a small amount of rhBMP-2 on the external surface and showed an initial burst release of rhBMP-2. The sustained release of rhBMP-2 from MCS-15 could continue promoting the proliferation and differentiation of hMSCs in vitro. It can be suggested that the MCS-15 possessed larger mesopore size and higher pore volume, which were the two key factors for the high loading and sustained release of rhBMP-2. It is expected that the MCS-15 could be a promising carrier material for loading and sustained release of bioactive macromolecules that could be used to enhance osteogenic properties, and MCS-15/rhBMP-2 system might be used as bone repair material with built-in osteoinduction function in bone reconstruction.

\section{Acknowledgments}

This study was supported by grants from the National Natural Science Foundation of China (Numbers 81271705 , 31271031 ), the international cooperation project of Ministry of Science and Technology of China (Number 2013DFB50280), the Major Program of Natural Science Foundation of Shanghai, People's Republic of China (Number 11JC1416302), Nano Special Program of Science and Technology Development of Shanghai (Number 12nm0500400), and Key Medical Program of Science and Technology Development of Shanghai (Numbers 12441903600, 12441902802).

\section{Disclosure}

The authors report no conflicts of interest in this work.

\section{References}

1. Lee EJ, Jun SH, Kim HE, Kim HW, Koh YH, Jang JH. Silica xerogelchitosan nano-hybrids for use as drug eluting bone replacement. J Mater Sci Mater Med. 2010;21:207-214.

2. Li X, Zhang L, Dong X, Liang J, Shi J. Preparation of mesoporous calcium doped silica spheres with narrow size dispersion and their drug loading and degradation behavior. Micropor Mesopor Mat. 2007;102: $151-158$.

3. Wang S. Ordered mesoporous materials for drug delivery. Micropor Mesopor Mat. 2009;117:1-9.

4. Manzano M, Vallet-Regi M. New developments in ordered mesoporous materials for drug delivery. J Mater Chem. 2010;20:5593-5604.

5. Yang P, Gai S, Lin J. Functionalized mesoporous silica materials for controlled drug delivery. Chem Soc Rev. 2012;41:3679-3698.

6. Dai C, Liu C, Wei J, Hong H, Zhao Q. Molecular imprinted macroporous chitosan coated mesoporous silica xerogels for hemorrhage control. Biomaterials. 2010;31:7620-7630.

7. Ariga K, Vinu A, Yamauchi Y, Ji Q, Hill JP. Nano architectonics for mesoporous materials. Bull Chem Soc Jpn. 2012;85:1-32.

8. Zhang Q, Liu F, Nguyen KT, et al. Multifunctional mesoporous silica nanoparticles for cancer-targeted and controlled drug delivery. $A d v$ Funct Mater. 2012;22:5144-5156.

9. Miyazono K, Kamiya Y, Morikawa M. Bone morphogenetic protein receptors and signal transduction. J Biochem. 2010;147:35-51.

10. Wang CK, Ho ML, Wang GJ, et al. Controlled-release of rhBMP-2 carriers in the regeneration of osteonecrotic bone. Biomaterials. 2009;30: $4178-4186$.

11. Shi S, Cheng X, Wang J, Zhang W, Peng L, Zhang Y. RhBMP-2 microspheres-loaded chitosan/collagen scaffold enhanced osseointegration: an experiment dog. J Biomater Appl. 2009;23:331-346.

12. Ong KL, Villarraga ML, Lau E, Carreon LY, Kurtz SM, Glassman SD. Off-label use of bone morphogenetic proteins in the United States using administrative data. Spine. 2010;35:1794-1800.

13. Ong KL, Villarraga ML, Lau E, Carreon LY, Kurtz SM, Glassman SD Expanded indication for recombinant human bone morphogenetic protein 2. Spine. 2011;36:1817-1818.

14. Kim J, Hollinger JO. Recombinant human bone morphogenetic protein-2 released from polyurethane-based scaffolds promotes early osteogenic differentiation of human mesenchymal stem cells. Biomed Mater. 2012;7:045008. 
15. Vivero-Escoto JL, Slowing II, Trewyn BG, Lin VSY. Mesoporous silica nanoparticles for intracellular controlled drug delivery. Small. 2010; 6:1952-1967.

16. Mieszawska AJ, Fourligas N, Georgakoudi I, et al. Osteoinductive silksilica composite biomaterials for bone regeneration. Biomaterials. 2010; 31:8902-8910.

17. Kempen DH, Lu L, Heijink A, et al. Effect of local sequential VEGF and BMP-2 delivery on ectopic and orthotopic bone regeneration. Biomaterials. 2009;30:2816-2825.

18. Shen H, Hu X, Yang F, Bei J, Wang S. An injectable scaffold: rhBMP-2-loaded poly(lactide-co-glycolide)/hydroxyapatite composite microspheres. Acta Biomater. 2010;6:455-465.

19. Luxenhofer R, Schulz A, Roques C, et al. Doubly amphiphilic poly (2-oxazoline) s as high-capacity delivery systems for hydrophobic drugs. Biomaterials. 2010;31:4972-4979.
20. Ishihara S, Matsumoto T, Onoki T, Uddin MH, Sohmura T, Nakahira A. Regulation of the protein-loading capacity of hydroxyapatite by mercaptosuccinic acid modification. Acta Biomater. 2010;6:830-835.

21. LanLevengood SK, Polak SJ, Poellmann MJ, et al. The effect of BMP-2 on micro- and macroscale osteointegration of biphasic calcium phosphate scaffolds with multiscale porosity. Acta Biomater. 2010;6: 3283-3291.

22. Hong Y, Chen X, Jing X, et al. Preparation, bioactivity, and drug release of hierarchical nanoporous bioactive glass ultrathin fibers. Adv Mater. 2010;22:754-758.

23. Crouzier T, Sailhan F, Becquart P, Guillot R, Logeart-Avramoglou D, Picart C. The performance of BMP-2 loaded TCP/HAP porous ceramics with a polyelectrolyte multilayer film coating. Biomaterials. 2011; 32:7543-7554.

\section{Publish your work in this journal}

The International Journal of Nanomedicine is an international, peerreviewed journal focusing on the application of nanotechnology in diagnostics, therapeutics, and drug delivery systems throughout the biomedical field. This journal is indexed on PubMed Central, MedLine, CAS, SciSearch ${ }^{\circledR}$, Current Contents ${ }^{\circledR} /$ Clinical Medicine,
Journal Citation Reports/Science Edition, EMBase, Scopus and the Elsevier Bibliographic databases. The manuscript management system is completely online and includes a very quick and fair peer-review system, which is all easy to use. Visit http://www.dovepress.com/ testimonials.php to read real quotes from published authors. 